\title{
Modeling and simulation of Positron Emission Mammography (PEM) based on double-sided CdTe strip detectors
}

\author{
I. Ozsahin ${ }^{a, 1}$ and M.Z. Unlu ${ }^{b}$ \\ ${ }^{a}$ Institute of Basic and Applied Sciences, Physics Department, Cukurova University, \\ Adana, Turkey \\ ${ }^{b}$ Izmir Institute of Technology, Electrical and Electronics Engineering Department, \\ Izmir, Turkey \\ E-mail: iozsahin@cu.edu.tr
}

ABSTRACT: Breast cancer is the most common leading cause of cancer death among women. Positron Emission Tomography (PET) Mammography, also known as Positron Emission Mammography (PEM), is a method for imaging primary breast cancer. Over the past few years, PEMs based on scintillation crystals dramatically increased their importance in diagnosis and treatment of early stage breast cancer. However, these detectors have significant limitations like poor energy resolution resulting with false-negative result (missed cancer), and false-positive result which leads to suspecting cancer and suggests an unnecessary biopsy.

In this work, a PEM scanner based on CdTe strip detectors is simulated via the Monte Carlo method and evaluated in terms of its spatial resolution, sensitivity, and image quality. The spatial resolution is found to be $\sim 1 \mathrm{~mm}$ in all three directions. The results also show that CdTe strip detectors based PEM scanner can produce high resolution images for early diagnosis of breast cancer.

KEYWORDS: Solid state detectors; Computerized Tomography (CT) and Computed Radiography (CR); Gamma camera, SPECT, PET PET/CT, coronary CT angiography (CTA); X-ray mammography and scinto- and MRI-mammography

\footnotetext{
${ }^{1}$ Corresponding author.
} 


\section{Contents}

1 Introduction 1

2 Detector design and simulation $\quad 3$

3 Image reconstruction $\quad 4$

3.1 Filtered back-projection (FBP) 4

4 Results 4

4.1 Spatial resolution 5

4.2 Sensitivity 5

4.3 Image quality 6

4.4 Derenzo Phantom 7

5 Conclusion $\quad 7$

\section{Introduction}

Molecular imaging of breast has been developed over the last few decades for the early diagnosis and treatment of breast cancer [1]. Although X-ray mammography is a widely accepted screening method for breast cancer because of its capability of detecting nonpalpable lesions, broad availability and low cost, an adjunct technique like PET for the functional imaging of tumor metabolism is needed [2]. The emergence of PET as a functional imaging modality of choice for diagnosis, staging, therapy monitoring, and assessment of recurrence in cancer has led to increasing demand for this advanced imaging technology. Functional imaging modalities like PET may provide an earlier diagnosis and more accurate staging than conventional anatomic imaging such as X-ray mammography, Computed Tomography (CT), and Magnetic Resonance Imaging (MRI). PET can be used in detection and characterization of primary breast cancers, in locoregional and systemic tumor staging and restaging, and in predicting and assessing tumor response to therapy.

Early diagnosis is widely acknowledged as being crucial in the successful treatment of breast cancer [3]. By detecting breast cancer at an early stage, women are not only more likely to survive, but they are also more likely to receive less aggressive treatments. Whole body (WB) PET scanners for breast cancer imaging have been used for many years [4]. The standard way of using PET for breast screening is to perform a WB scan. It has also been shown to be of great value in the evaluation of the response to therapy and in the detection of recurrent breast cancer. In fact, due to its limited spatial resolution, PET is not able to detect lesions of less than $1 \mathrm{~cm} \mathrm{[6].} \mathrm{PET} \mathrm{works}$ based on the premise that malignant cells have high metabolic rates and take up more glucose than the surrounding tissue [7]. Due to the fact that malignant cells express more specific transporter proteins with a greater affinity for glucose than those expressed by normal cells, glucose uptake is 
enhanced in malignant cells [7]. Malignant cells have also been shown to have an overall increased number of glucose transporters [8]. Since fluorodeoxyglucose (FDG or ${ }^{18}$ F-FDG) is taken up like glucose but is not metabolized, it becomes metabolically trapped within the cancer cell [9]. In principle, PET is able to detect malignant lesions with high sensitivity, but breast cancer detection also requires the ability to demonstrate nonpalpable, small (less than $1.0 \mathrm{~cm}$ ) invasive, and in situ malignancies. Unfortunately, WB PET does not have all these capabilities and thus PET is not used for primary breast cancer detection [10]. Thus, alternative solutions to the WB PET scanners have been searched for many years. PEM is an emerging technology that allows high diagnostic accuracy and high resolution for non-invasive breast cancer detection [11]. PEM devices are promising imaging devices with their compact design that enables compression of the breast between the paddles gently. By limiting the field of view (FOV) to the body part to be examined, these cameras can achieve very high spatial resolution as well as very high sensitivity and specificity. Another reason for the emerging of PEM is that conventional mammography has low positive predictive value especially in the case of women with mammographically dense breast.

Currently, a few research centers are evaluating dedicated PEM devices that may potentially improve identification of small breast cancers [11]. Sensitivity, image reconstruction, and cost are taken into account when choosing PEM geometry. There are several different geometric designs such as rectangular geometry (four detector plates surrounding the breast [12]), ring geometry [13], and coplanar geometry [11]. The coplanar detector geometry based on scintillation crystals is the most used, since it provides great spatial resolution in plane ${ }^{1}$ down to $2 \mathrm{~mm}$ full width at half maximum (FWHM) [11] and high contrast images with $4 \mathrm{~mm}$ minimum detectable lesion size for 10:1 tumor to normal tissue ratio [14]. The detector heads are positioned in opposing fashion on either side of the body part and move in parallel across the paddles during a scan [11]. However, scintillation crystals based detectors have a lot of intrinsic limitations since they are incompatible with strong magnetic fields of the MRI if used with the photomultiplier tubes (PMTs), they have relatively poor energy resolution (5-10\% for commonly used crystals [15]), and relatively large parallax error due to the uncertainty of depth of interaction information ( $>5 \mathrm{~mm}$ FWHM) [16]. All these intrinsic limitations dramatically affect the detector sensitivity and image quality, and put a serious limitation on the minimum detectable tumor size and on the correct assessment of the malignancy of small lesions, regardless of the chosen geometry [17]. This is a consequence of the partial volume effect [18] that produces a loss of intensity and a smearing of the activity distribution around those high uptake regions whose volume is smaller than twice the detector resolution. In the particular case of coplanar scanners, it implies a resolution a factor of 4 worse than the inplane spatial resolution: a large parallax error results in a dramatic deterioration of the resolution up to $8 \mathrm{~mm}$ FWHM along the vertical axis [14]. Obviously, all these pose constraints on the minimum detectable tumor size and on the correct assessment of malignancy of small lesions which are probably the two most important factors to determine the effectiveness of any breast cancer treatment [17]. In this study, a high resolution PEM camera based on CdTe strip detectors is proposed to overcome the aforementioned limitations of PEM scanners based on scintillating crystals. The proposed detector was modeled and a performance evaluation in terms of sensitivity, spatial resolution, and image quality was performed. The detector was tested following the prescriptions

\footnotetext{
${ }^{1}$ Parallel to the paddle surface.
} 


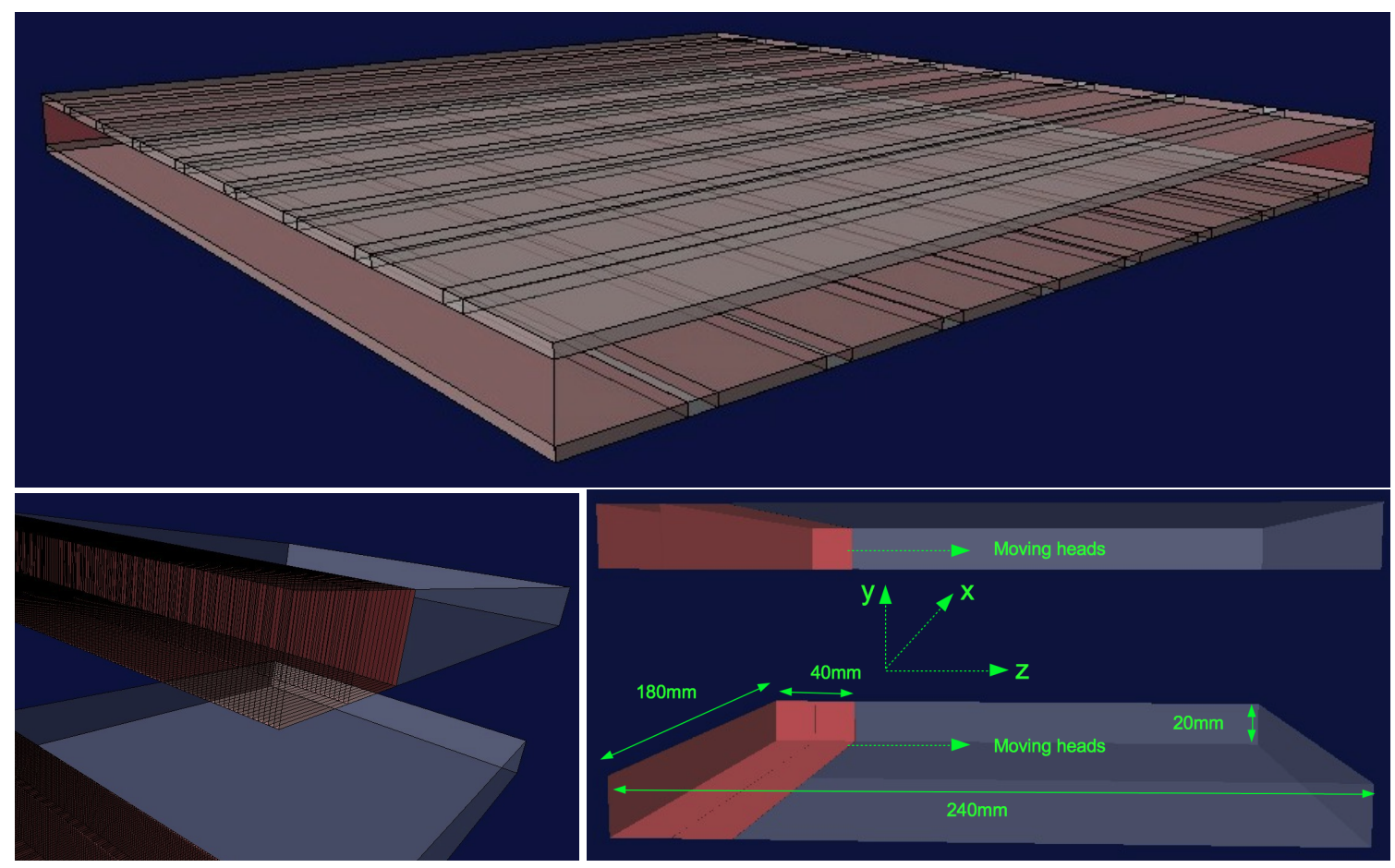

Figure 1. A part of 9x9 of unit module of the detector (top), two lines of 180 successive arrays of such a unit (bottom-left), the full detector with the chosen axes (bottom-right).

of the National Electrical Manifacturers Association (NEMA) Standards Publication NU 4-2008: Performance Measurements of Small Animal Positron Emission Tomographs [19].

\section{Detector design and simulation}

The proposed PEM scanner consists of orthogonally aligned double-sided CdTe strip detectors. The unit detector has $1 \mathrm{~mm}$ thickness and 40 strip electrodes with a $0.4 \mathrm{~mm}$ width and $0.1 \mathrm{~mm}$ gaps in between on each side. Two lines of 180 successive arrays of unit detectors constitute the detector head. The detector (figure 1 ) is able to detect incoming gamma rays by the thin edge of the unit module and provides a $1.0 \times 0.5 \mathrm{~mm}^{2}$ effective pixel size $(1.0 \mathrm{~mm}$ for the thickness of the module and $0.5 \mathrm{~mm}$ for the strip). Two heads are positioned in opposing fashion in a way to move parallely, with a paddle separation of $65 \mathrm{~mm}$ (figure 1 (bottom-right)). Platinum $(\mathrm{Pt}$ ) as cathode and Aluminum (Al) as anode can be used as segmented strips [20]. The total number of channels is 2 heads $\times 2$ lines $\times 180$ detectors $\times 80$ channels $=57600$. Each unit detector can be mounted on a kapton foil with a $50 \mu \mathrm{m}$ thickness. The CdTe detectors are able to couple to ceramic fan-out board with bump bonding or flip-chip process. Then wire bonding can be done from Application Specific Integrated Circuit to the electrode of the ceramic board [20]. Readout of the detectors can be achieved by using a double-sided flexible circuit with thickness of $100 \mu \mathrm{m}$, which allows for a good packing fraction for the sensitive detector material [21].

The Monte Carlo (MC) simulation for the evaluation of the proposed design was carried out within the framework of Geant4-based Architecture for Medicine-Oriented Simulations (GAMOS) [22]. 

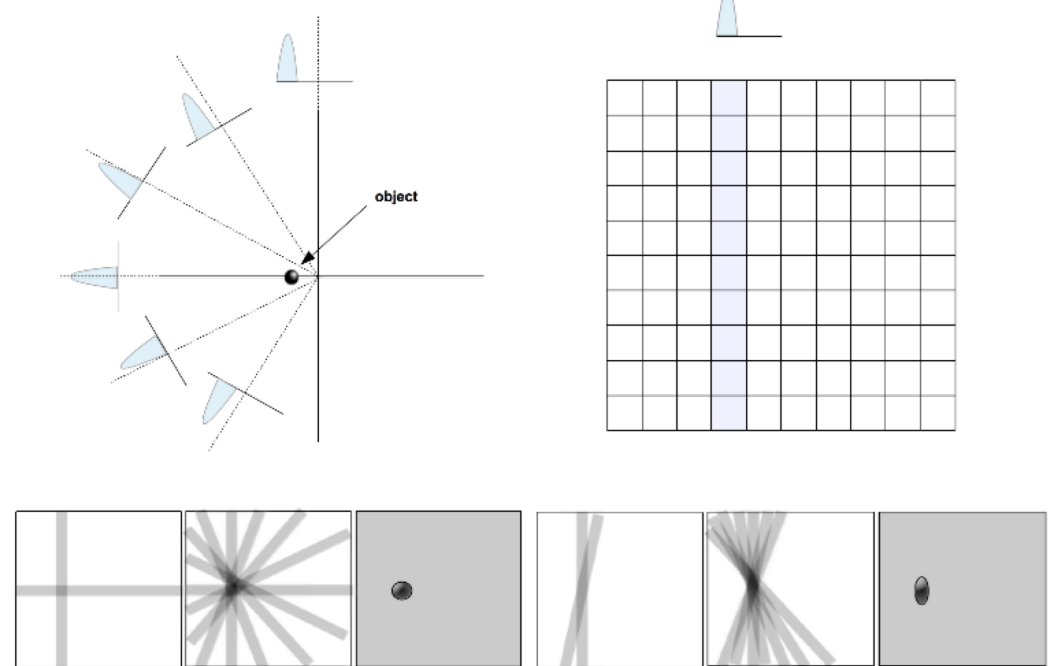

Figure 2. Illustration of the back-projection. Projection profiles for a point source for different projection angles (top-left), back-projection of one profile across the image at $0^{\circ}$ angle (top-right), back-projection after 2 angles, 8 angles and all angle projections, from left to right, respectively (bottom-left), and backprojections in case of PEM (bottom-right).

\section{Image reconstruction}

\subsection{Filtered back-projection (FBP)}

FBP is the most well-known method for image reconstruction and the most used algorithm in PET. The first step of the FBP method is to acquire projection profiles at $N$ projection angles. Then the algorithm computes the 1D Fourier transform (FT) of all projections. After computing 1D FT, a combination of a high-pass filter and low-pass filter is applied to each profile. While high-pass filter is needed to reduce the star artifact resulting from back-projection, low-pass filter is needed to reduce the contribution of high-frequency noise (remained after the high-pass filter) and smooth the image. After the filtering, the inverse FT of each profile is computed to obtain a modified projection profile. The last step is to perform conventional back-projection using the filtered profiles. Figure 2 illustrates the back-projection algorithm.

Even though FBP is very fast, exact (it only involves a single back-projection step), and requires less computational time with respect to iterative methods, it may produce artifacts in the image obtained especially when the detector has a coplanar geometry. Since a PEM scanner has limited angular coverage, some angular projections are missing. Hence, the reconstructed image will be spoiled by artifacts in particular for objects far from the centre of the detector (see figure 2 bottom-right). Also this effect can ben seen in the results of spatial resolution test, in the following section.

\section{Results}

The evaluation of the performances was carried out by following the NEMA NU 4-2008 indications. 


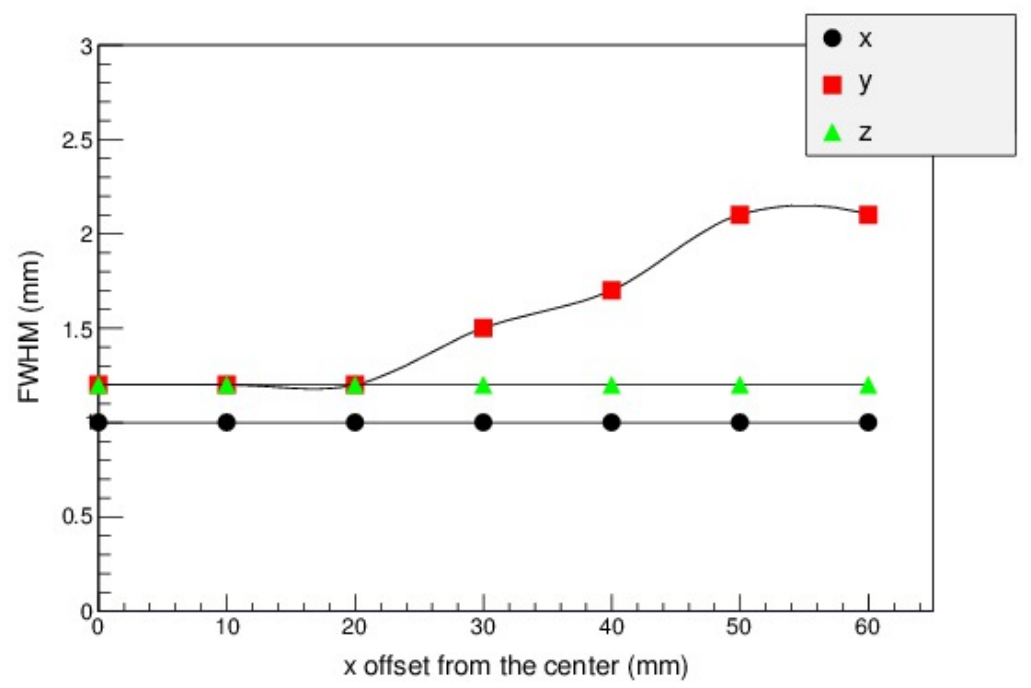

Figure 3. Spatial Resolution with FBP.

\subsection{Spatial resolution}

The purpose of this test is to characterize the widths of the point spread functions (PSF) from the reconstructed image of compact radioactive sources. The radionuclide used for this measurement was ${ }^{22} \mathrm{Na}$ with an activity less than that at which the random coincidence rate exceeds $5 \%$ of the total event rate. For this test, a point-like source of $0.15 \mathrm{~mm}$ radius with an activity of $20.4 \mathrm{kBq}$ embedded in an acrylic cube with a side of $10 \mathrm{~mm}$ was used. The test was performed by placing the point like source in the $\mathrm{x}$-direction at 0 (center of the FOV), 10, 20, 30, 40, 50, and $60 \mathrm{~mm}$. Then the measurement was acquired for each position. 100,000 prompt coincidences were collected for each position. Figure 3 shows the FWHM of the PSF of the reconstructed image.

\subsection{Sensitivity}

The purpose of this test is to measure the sensitivity or ability of the scanner to detect positron annihilation gamma rays. The same point source as described in the previous section was used for this test. The source was first placed in the one edge of the FOV and scanned. Then it was stepped axially (along the $\mathrm{z}$ direction) in $2 \mathrm{~mm}$ increments to the other edge of the FOV and scanned after each step. As seen in figure 4, the sensitivity profile is uniform in the central region and it decreases as closing to the edge of the detector as expected. Sensitivity (cps/Bq) for each slice was calculated as follows:

$$
S_{i}=\frac{R_{i}-R_{B, i}}{A_{\text {cal }}}
$$

where $R_{i}$ is the source counting rate, $R_{B, i}$ is the background counting rate for acquisition i (taken as zero), and $A_{c a l}$ is the source activity. The total system sensitivity is $0.17 \mathrm{cps} / \mathrm{Bq}$ calculated as sum of the sensitivities over all the slices (eq. (4.2)).

$$
S_{\mathrm{tot}}=\sum_{\substack{a l l \\ i}} S_{i}
$$




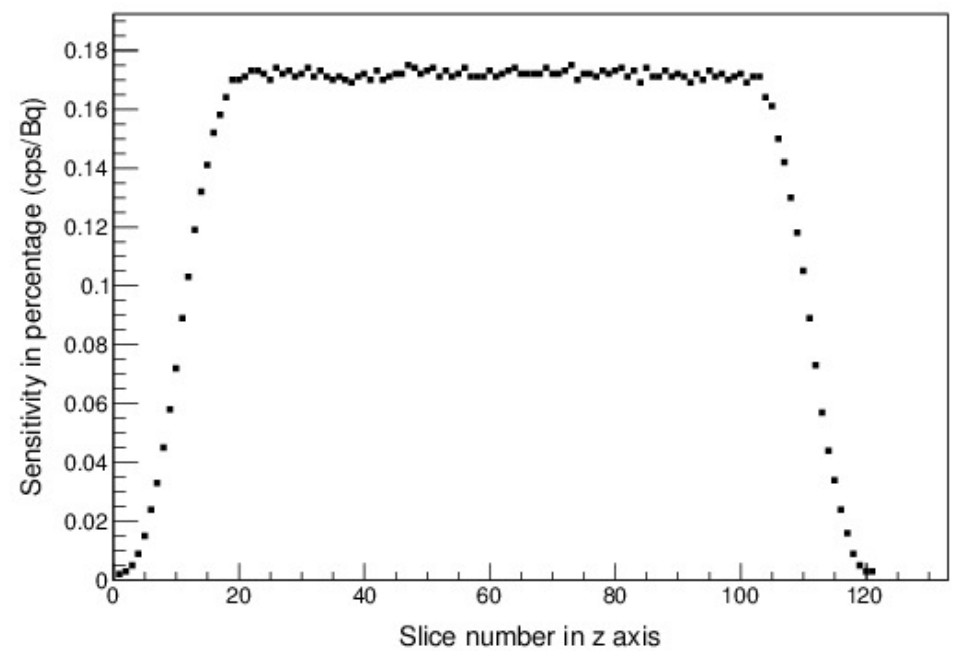

Figure 4. Sensitivity profile.
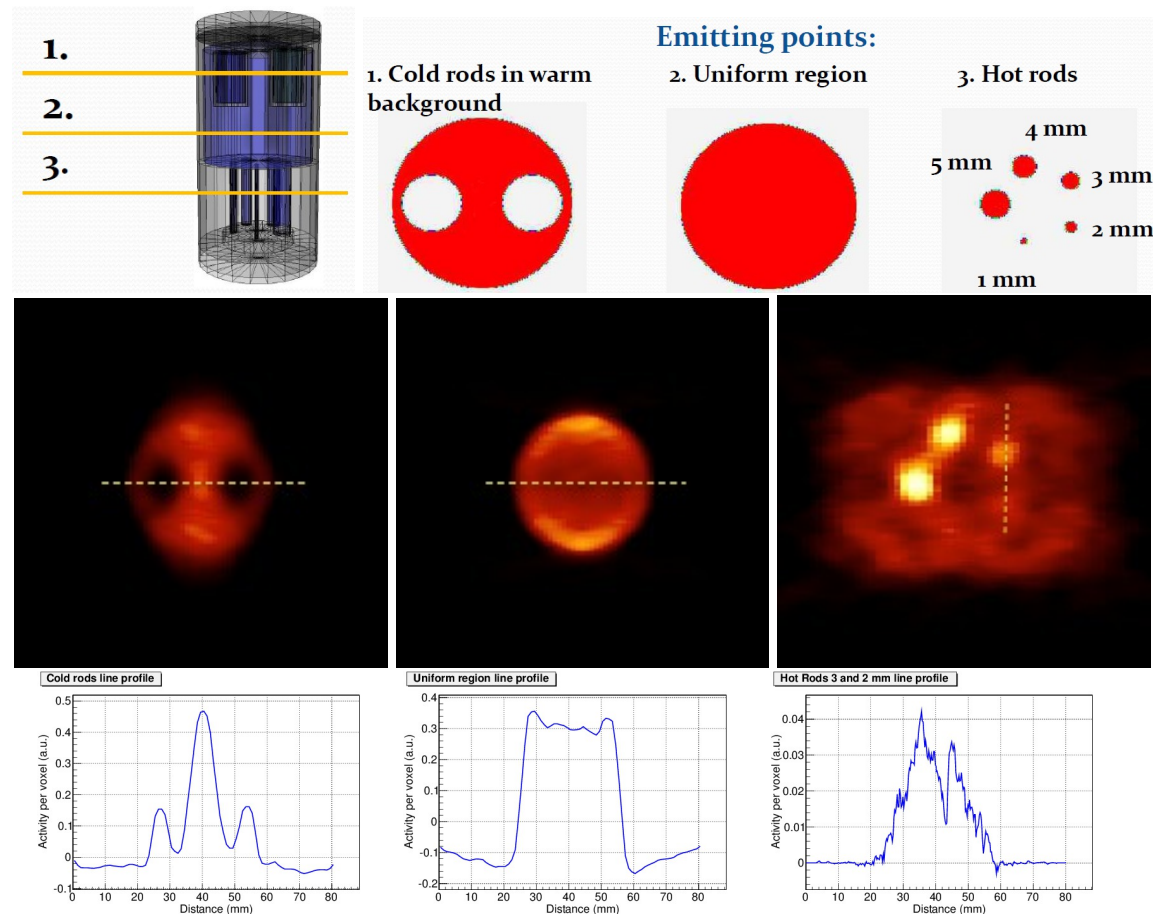

Figure 5. NEMA image quality phantom and cross-sectional images (top), activity distribution obtained with FBP for the cold rods in hot background, the uniform region and the hot rods, from left to right, respectively (center), corresponding line profiles (bottom).

\subsection{Image quality}

For the image quality evaluation, an image quality phantom, as defined in the NEMA NU4-2008 document is simulated. The phantom has central uniform region, cold rods in a hot background in the upper part and hot rods in a cold background in the lower part. The central uniform region, 


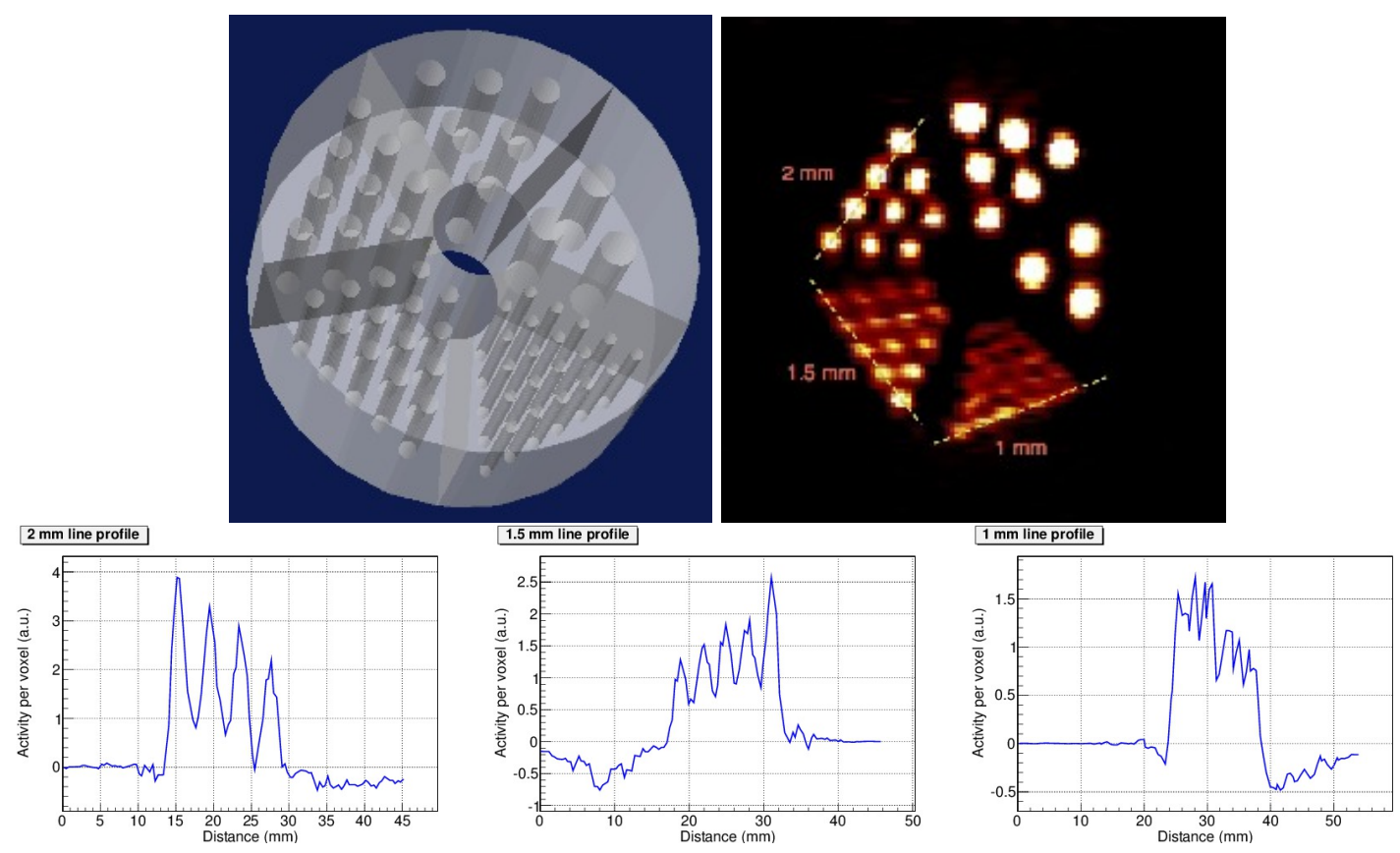

Figure 6. Image of the Derenzo Phantom with GAMOS (top-left), the cross-sectional reconstructed image of Derenzo Phantom with FBP (top-right), corresponding line profiles along the $2 \mathrm{~mm}, 1.5 \mathrm{~mm}$, and $1 \mathrm{~mm}$ rods, from left to right, respectively (bottom).

as well as the rods, are filled with ${ }^{18} \mathrm{~F}$ radioactive water of $10^{5} \mathrm{~Bq}$ total activity. The phantom is placed in the center of the FOV aligned vertically. A total of 1.5 million coincidences are collected. The paddle separation is $72 \mathrm{~mm}$ for this test. Figure 5 shows the image of the NEMA image quality phantom with cross sectional line profiles.

\subsection{Derenzo Phantom}

A Derenzo Phantom is simulated in addition to evaluate the performance. The Derenzo Phantom [23] is a cylinder is made of plexiglass with the inner radius of $4 \mathrm{~mm}$, the outer radius of 18 $\mathrm{mm}$ and the length of $15 \mathrm{~mm}$. The phantom has rods of different diameters from $1 \mathrm{~mm}$ to $5 \mathrm{~mm}$ as it is seen in figure 6 . The rods are filled with ${ }^{18} \mathrm{~F}$ radioactive water of $10^{5} \mathrm{~Bq}$ total activity. In the image of the Derenzo Phantom (see figure 6) obtained with 1.3 million coincidences, the rods with the diameter of $1.5 \mathrm{~mm}$ can be seen clearly and well separated from the neighboring rods, and the corresponding line profile exhibits a 2:1 Peak-to-Valley ratio.

\section{Conclusion}

The proposed PEM design is evaluated via Monte Carlo simulation. Several tests are performed to assess the imaging performance of the PEM scanner with the accurate modeling of the required experimental conditions. The image quality performance of the simulated PEM system has been characterized using NEMA Standards Publication NU 4-2008. Images have been reconstructed with FBP algorithm with no correction applied. Even though FBP is fast, it creates artifacts in the final image due to the limited angular coverage. Simulation results show the feasibility and 
capability for providing metabolic images with a sensitivity of $0.17 \mathrm{cps} / \mathrm{Bq}$, comparable with the published values for the state-of-the-art commercial scanners [11]. This work also shows that the proposed PEM scanner can achieve an excellent image resolution as low as $\sim 1 \mathrm{~mm}$ FWHM in all the three directions for the point like source. The resolution is found around $2-3 \mathrm{~mm}$ for more realistic circumstances.

\section{References}

[1] C.B. Hruska et al., Scientific Impact Recognition Award: Molecular breast imaging: A review of the Mayo Clinic experience, Am. J. Surg. 196 (2008) 470.

[2] A.Y. Rostom et al., Positron emission tomography in breast cancer: a clinicopathological correlation of results, British J. Radiol. 72 (1999) 1064.

[3] D.E. Henson and L.A. Riess, Progress in early breast cancer detection, Cancer 65 (1990) 2155; Lancet Oncol 9 (2008) 730.

[4] F. Benard and E. Turcotte, Imaging in breast cancer: Single-photon computed tomography and positron-emission tomography, Breast Cancer Res. 7 (2005) 153.

[5] A.M. Wallace, C. Comstock, C.K. Hoh and D.R. Vera, Breast imaging: a surgeon's prospective, Nucl. Med. Biol. 32 (2005) 781.

[6] W.B. Eubank and D.A. Mankoff, Evolving role of positron emission tomography in breast cancer imaging, Semin. Nucl. Med. 35 (2005) 84.

[7] H.S. Lim et al., FDG PET/CT for the detection and evaluation of breast diseases usefulness and limitations, RadioGraphics 27 (2007) 197.

[8] C. Love, M.B. Tomas, G.G. Tronco and C.J. Palestro, FDG PET of infection and inflammation, Radio- Graphics 25 (2005) 1357.

[9] H. Minn and I. Soini, ${ }^{18}$ F fluorodeoxyglucose scintigraphy in diagnosis and follow up of treatment in advanced breast cancer, Eur. J. Nucl. Med. 15 (1989) 61.

[10] R. Kumar et al., Clinicopathologic factors associated with false negative FDG-PET in primary breast cancer, Breast Cancer Res Treat 98 (2006) 267.

[11] W. Luo, E. Anashkin and C.G. Matthews, Performance Evaluation of a PEM Scanner Using the NEMA NU 4-2008 Small Animal PET Standards, IEEE Trans. Nucl. Sci. 57 (2010) 94.

[12] G.C. Wang, J.S. Huber, W.W. Moses, J. Qi and W.-S. Choong, Characterization of the LBNL PEM camera, IEEE Trans. Nucl. Sci. 53 (2006) 1129.

[13] L. Moliner et al., Design and evaluation of the MAMMI dedicated breast PET, Med. Phys. 39 (2012) 5393.

[14] L. MacDonald et al., Clinical imaging characteristics of the positron emission mammography camera: PEM Flex Solo II, J. Nucl. Med. 50 (2009) 1666.

[15] G.F. Knoll, Radiation Detection and Measurements, John Wiley and Sons Inc., New York (2010).

[16] C.W. Lerche et al., Dependency of Energy, Position and Depth of Interaction Resolution on Scintillation Crystal Coating and Geometry, IEEE Trans. Nucl. Sci. 55 (2008) 1344.

[17] Breast Cancer Facts and figures 2011-2012, American Cancer Society (2012).

[18] G.B. Saha, Basics of PET Imaging, Springer Science and Business Media Inc., New York (2005). 
[19] National Electrical Manufacturers Association, Performance Measurements of Small Animal Positron Emission Tomographs, National Electrical Manufacturers Association Rosslyn, VA (2008).

[20] S. Watanabe et al., High energy resolution hard X-ray and gamma-ray imagers using CdTe diode devices, IEEE Trans. Nucl. Sci. 56 (2009) 777.

[21] G.S. Mitchell et al., CdTe Strip Detector Characterization for High Resolution Small Animal PET IEEE Trans. Nucl. Sci. 55 (2008) 870.

[22] P. Arce et al., Gamos: A framework to do Geant4 simulations in different physics fields with an user-friendly interface, Nucl. Instrum. Meth. A 735 (2014) 304.

[23] T.F. Budinger, S.E. Derenzo, G.T. Gullberg, W.L. Greenberg and R.H. Huesman, Emission computer assisted tomography with single-photon and positron annihilation photon emitters, J. Comput. Assist Tomogr. 1 (1977) 131. 